\title{
Left Bundle Branch Pacing Guided by Premature Ventricular Complexes During Implant
}

\author{
Shunmuga Sundaram ${ }^{1}$ and Pugazhendhi Vijayaraman ${ }^{2}$ \\ ${ }^{1}$ Velammal Medical College Hospital and Research Institute \\ ${ }^{2}$ Geisinger Heart institute
}

June 9, 2020

\begin{abstract}
Left bundle branch pacing (LBBP) provides synchronized contraction of ventricles and overcomes the limitation of His bundle pacing (HBP). The pacing lead is placed deep inside the septum 1-1.5 cm apical to the distal His bundle region. The criteria for capture of left bundle branch (LBB) have been proposed but it has never been validated. LBB potentials may not be demonstrable in all patients. Premature ventricular complexes (PVC) are often noted while positioning the lead in the interventricular septum. The morphology of the PVCs depends on the depth of the lead in the septum. We describe a novel method for lead placement to capture LBB by monitoring the morphology and duration of PVCs in four patients with different pacing indications. Rapid rotations were stopped immediately on observing a PVC with narrow QRS duration and qR (right bundle branch delay) pattern in lead V1. LBB potential and non-selective to selective LBB capture could be demonstrated after placing the lead. PVC guided lead placement would help in final positioning of the lead, avoid perforation into left ventricle cavity and to confirm conduction system capture.
\end{abstract}

\section{Title Page}

INNOVATIVE TECHNIQUES

Left Bundle Branch Pacing Guided by Premature Ventricular Complexes During Implant

\section{Author Details}

Dr. Shunmuga Sundaram Ponnusamy MD., DM., PDF (EP)

Assistant Professor, Department of Cardiology

Velammal Medical college hospital and research institute

Madurai, Tamilnadu, India

Dr. Pugazhendhi Vijayaraman, MD., FHRS Professor of Medicine, Geisinger Commonwealth School of Medicinie Geisinger Heart Institute, MC 36-10 1000 E Mountain Blvd, Wilkes-Barre, PA 18711

Disclosures:

SSP: None

PV: Honoraria, consultant, research, fellowship support: Medtronic, consultant: Boston

Scientific, Abbott, Biotronik, Eaglepoint LLC

Key Words: Left bundle pacing, premature ventricular complex, heart failure, AV junction ablation Word count: 1194 words 


\section{INNOVATIVE TECHNIQUES}

Left Bundle Branch Pacing Guided by Premature Ventricular Complexes During Implant

\section{Introduction}

Physiological pacing has witnessed a rapid growth in the last decade. His bundle pacing (HBP) provides electrical and mechanical synchrony but limited by high pacing thresholds and lead revisions ${ }^{1}$. Left bundle branch pacing (LBBP) has recently been shown to be a promising alternative to HBP. Several criteria for left bundle capture have been proposed ${ }^{2}$, but needs to be validated. We describe premature ventricular complex (PVC) guided lead implantation as a novel approach to perform LBBP.

\section{Case 1:}

A 35 years old woman with non-ischemic cardiomyopathy (NICM), left bundle branch block (LBBB) and left ventricular (LV) dysfunction (ejection fraction 28\%) was referred for cardiac resynchronization therapy (CRT). After obtaining informed consent, LBBP was performed using C315 His sheath and 3830 Selectsecure $^{\mathrm{tm}}$ lead (Medtronic, Minneapolis, MN). The pacing lead was positioned deep inside the septum $1.5 \mathrm{~cm}$ apical to the distal His bundle region by 4-5 rapid turns. PVCs with changing morphology were noted during lead advancement. Rotation was stopped immediately on observing a PVC (VES1) with narrow QRS duration and qR (right bundle branch [RBB] delay) pattern in lead V1 (Figure 1A). No potentials were noted during baseline LBBB rhythm. Non-selective to selective capture of left bundle branch (LBB) could be demonstrated by change in QRS morphology and discreet local ventricular electrogram at near threshold output (Figure 1C). Pacing threshold was $0.4 \mathrm{~V}$ at $0.5 \mathrm{~ms}$ and unipolar pacing impedance of 670 ohms. LBB paced QRS morphology mimicked VES1 with duration of $122 \mathrm{~ms}$ and peak left ventricular activation time (pLVAT) in lead V5 of 65ms (Figure 1B). AV interval was optimized to correct the RBB delay (Figure 1D).
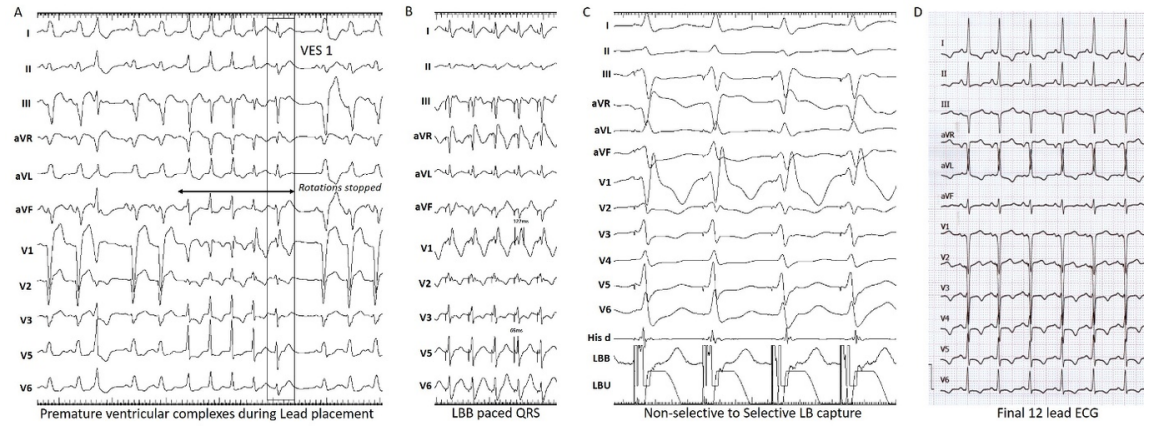

Figure 1: Left bundle branch pacing for NICM with LBBB. A - PVCs with changing morphology from $Q S$ to qR pattern (VES1) in lead V1 noted during rapid rotation. B - LBB paced QRS morphology mimicked VES1 with duration of 122ms and pLVAT of 65ms. C-Non selective (first 2 beats) to selective $L B B$ capture (last 2 beats) at near threshold output. D- Final 12 lead electrocardiography (ECG) after RBB delay correction by optimizing the $A V$ delay (QRS duration 98ms). His d-distal His bundle electrogram, $L B B$ and $L B U-$ Pacing lead electrogram bipolar and unipolar respectively

\section{Case 2:}

A 72 years old man was referred for the management of permanent atrial fibrillation with uncontrolled ventricular rates and LV dysfunction. Echocardiography showed moderate mitral regurgitation with LV ejection fraction of $32 \%$. Atrioventricular junction (AVJ) ablation with physiological pacing option was recommended. LBBP was attempted as previously described. PVCs of changing morphology were noted while placing the lead deep inside the septum. Rotation was stopped immediately after observing PVC (VES2) with narrow QRS duration and $\mathrm{qR}$ in lead V1 (Figure 2A). Non-selective to selective capture of LBB could be demonstrated at near threshold output (Figure 2D). LBB paced QRS mimicked VES2 with 
duration of $124 \mathrm{~ms}$ and pLVAT of $65 \mathrm{~ms}$ (Figure $2 \mathrm{~B}$ ). The pacing threshold was $0.6 \mathrm{~V} / 0.5 \mathrm{~ms}$ and lead impedance of $730 \mathrm{ohms}$. LBB potential was recorded on the LBBP lead electrogram (LBB-ventricular interval of $25 \mathrm{~ms}$, Figure $2 \mathrm{C}$ ). AVJ ablation was completed using an irrigated-tip ablation catheter.
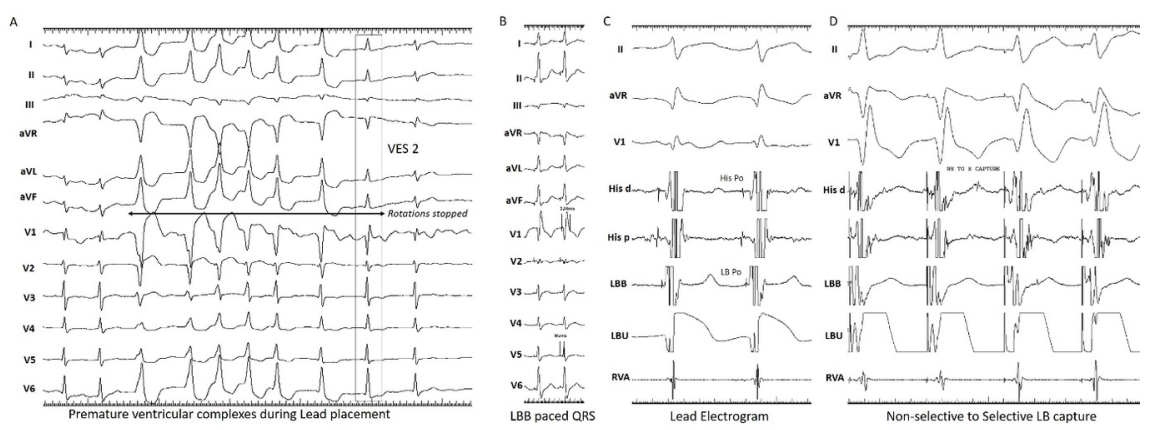

Figure 2: $\mathbf{L B B P}$ and $\boldsymbol{A} \boldsymbol{V} \boldsymbol{J}$ ablation. $A-P V C s$ with changing morphology during lead rotation. B- LBB paced QRS mimicked VES2 with duration of 124ms and pLVAT 65ms. C-Pacing lead electrogram (LBB) showing sharp LBB potentials ( $L B$ Po) preceding the local ventricular electrogram. $D-$ Non selective (first 2 beats) to selective (last 2 beats) capture of $L B B$. His $d$ and $p$ - His distal and proximal electrogram, LBB and LBU - Pacing lead electrogram bipolar and unipolar, RVA - right ventricular electrogram, His Po - His bundle potential

\section{Case 3:}

A 65-year-old woman with NICM, LBBB and LV dysfunction (ejection fraction 30\%) was referred for CRT. LBBP was performed using C315His sheath and 3830 Selectsecure $^{\mathrm{tm}}$ lead. While positioning the lead deep inside the septum, PVCs with changing morphology were noted. Rotation was stopped immediately on observing PVC (VES3) with narrow QRS duration and qR in lead V1 (Figure 3A). No potentials were noted during baseline LBBB rhythm. The pacing threshold was $0.3 \mathrm{~V}$ at $0.5 \mathrm{~ms}$ and pacing impedance of $580 \mathrm{ohms}$. The final LBB paced QRS morphology mimicked VES3 (Figure 3B) with duration of 124ms (pLVAT - 78ms). $\mathrm{RBB}$ delay was corrected by optimizing the AV interval and pacing output (Figure 3C).
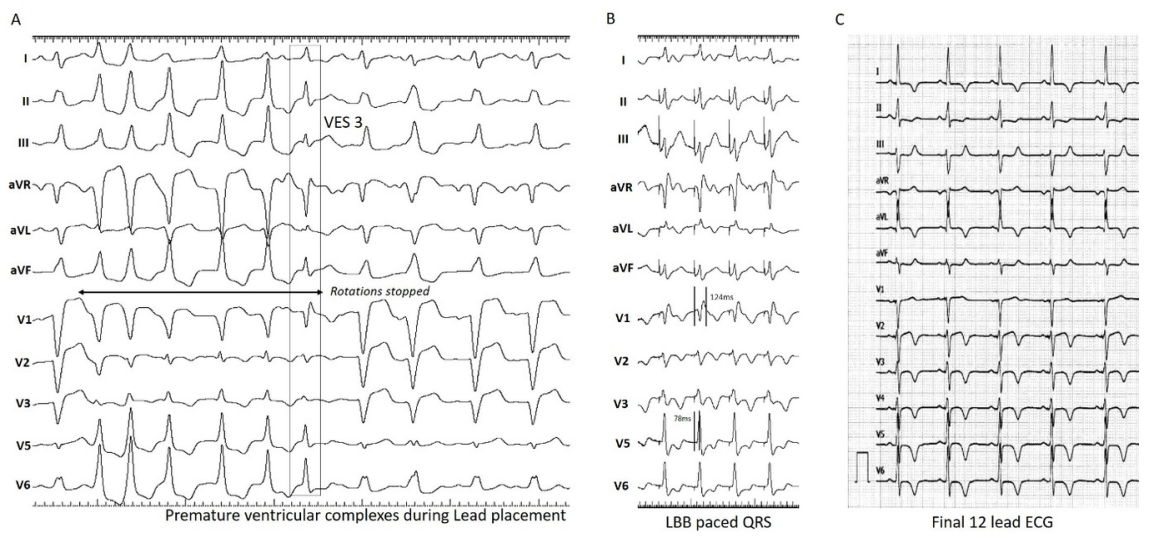

Figure3: $\mathbf{L B B P}$ for NICM with $\mathbf{L B B B}$. A - Rapid premature ventricular complexes during lead rotation. $B-L B B$ paced QRS mimicked VES4 with duration of $124 \mathrm{~ms}$ and pLVAT of $78 \mathrm{~ms}$. C - Final 12 lead ECG after correcting RBB delay by AV interval optimization (QRS duration 108ms)

\section{Case 4:}


A 73 years old woman with normal LV function presented with symptomatic complete heart block. LBBP was done using 3830 Selectsecure ${ }^{\mathrm{tm}}$ lead and C315His sheath. The pacing lead was positioned deep inside the septum by $4-5$ rapid turns. PVCs with changing morphology were noted during lead advancement. Lead rotation was stopped immediately after observing narrow complex PVC (VES4) with qR pattern in lead V1 (figure 4A). Electrograms from the pacing lead demonstrated sharp left bundle potential (Figure 4C) preceding the ventricular electrogram (LB-ventricular interval of $20 \mathrm{~ms}$ ). Pacing threshold was $0.4 \mathrm{~V}$ at $0.5 \mathrm{~ms}$ and lead impedance was $680 \mathrm{ohms}$. The paced QRS morphology mimicked the narrow PVC morphology (VES4) with QRS duration of $114 \mathrm{~ms}$ with pLVAT in lead V5 of 70ms (Figure 4B). RBB delay was corrected by optimizing pacing output to allow anodal capture (Figure 4D)
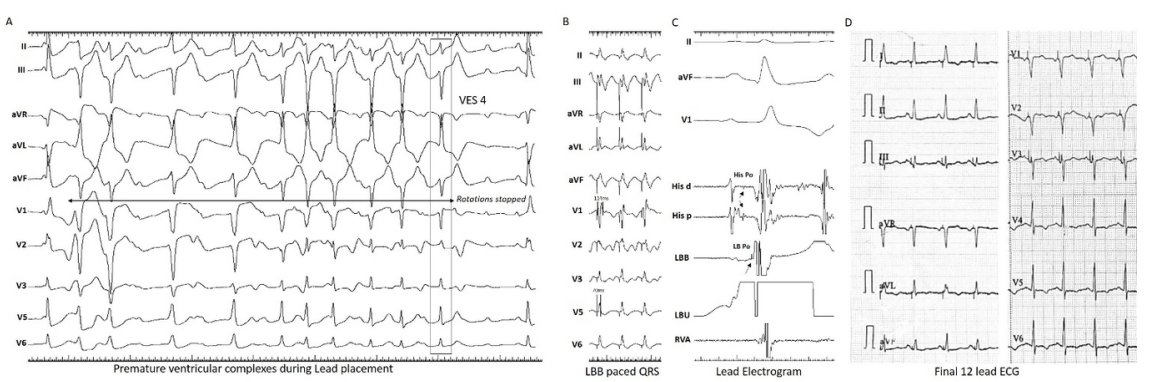

Figure 4: LBBP for Complete heart block. A - PVCs during LBB lead placement. B - Paced QRS morphology mimicked VES4 with duration of $114 \mathrm{~ms}$ and pLVAT of 70ms. C-Pacing lead electrogram showing sharp LBB potential ( $L B$ Po) preceding the local ventricular electrogram. $C$ - Final 12 lead ECG after RBB delay correction by anodal capture (QRS duration 100ms). His d and $p$ - His distal and proximal electrogram, $L B B$ and $L B U$ - Pacing lead electrogram bipolar and unipolar, RVA - right ventricular electrogram, His Po - His bundle potential

\section{Discussion:}

Left bundle branch pacing is a promising alternative to HBP. Presence of right bundle branch conduction delay pattern (qR in lead V1) and demonstration of LB potentials are often used as criteria for LBB capture ${ }^{2,3}$. LBB potentials may not be demonstrable in all patients, especially in those with LBBB. Transition in QRS morphology from non-selective to selective LB capture or nonselective to LV septal capture may be noted at near threshold outputs ${ }^{4}$. Rapid rotation of the pacing lead is necessary to achieve deep penetration of the interventricular septum. Perforation into the LV cavity can occur if the lead is advanced too rapidly. Premature ventricular complexes are commonly noted while positioning the lead in the interventricular septum. The PVC morphology depends on the depth of the lead in the septum. We observed gradual change in morphology from wide QRS with QS morphology in lead V1 to narrow QRS with qR pattern in lead V1 as the lead penetrated the septum from right ventricular side to the LBB area. In all four cases, rapid rotations were stopped as soon as PVCs with narrow QRS/qR pattern were observed (VES1, VES2, VES3 and VES4). Paced QRS morphology matched the PVC morphology with short and constant pLVAT at differential pacing (high and low output). Though the pacing indications varied in these patients PVC morphology predicted LB capture and guided in deciding the lead depth. LBB potentials were noted in two patients (CHB and AVJ ablation cases). It is possible to record LBB potentials in patients with LBBB during PVCs of RBB morphology if continuous recording can be performed during lead rotations. Lack of a revolving connectorpin during lead rotations is a limitation with the current implant technique. Further rotations were avoided, preventing perforation of septum. Monitoring the change in PVC morphology and QRS duration during lead fixation would help in final positioning of the LBB pacing lead and confirming conduction system capture.

Since the initial description of LBBP, multiple studies have shown the safety and efficacy of left bundle branch pacing. Huang et $\mathrm{al}^{5}$, demonstrated $97 \%$ success rate in LBBP for non-ischemic cardiomyopathy and LBBB along with significant improvement in LV ejection fraction at 1 year. A large retrospective multicenter 
study by Vijayaraman et $\mathrm{al}^{6}$, showed $85 \%$ success rate in achieving cardiac resynchronization therapy by LBBP (277 out of 325 patients). Improvement in LVEF was noted in both ischemic and non-ischemic cardiomyopathy and similarly in patients with LBBB and non-LBBB. Conduction system pacing combined with AV node ablation showed a high success rate in persistent atrial fibrillation patients with heart failure and ICD indication ${ }^{7}$. This study also showed significant improvement in LV function and reduction in inappropriate shocks.

LBBP is emerging as a promising option to deliver physiological pacing. Though several criteria have been proposed to confirm capture of left bundle, prospective studies are necessary to validate. PVC guided lead placement would help in final positioning of the lead and avoid septal perforation into LV cavity

\section{References}

1. Subzposh FA, Vijayaraman P. Long-term results of His bundle pacing. Card Electrophysiol Clin 2018;10:537-42.

2. Huang W, Chen X, Su L, et al. A beginner's guide to permanent left bundle branch pacing. Heart Rhythm 2019;16;1791-6

3. Vijayaraman P, Subzposh FA, Naperkowski A, et al. Prospective evaluation of feasibility, electrophysiologic and echocardiographic characteristics of left bundle branch area pacing. Heart Rhythm 2019;16:1774-1782

4. Vijayaraman P, Huang W. Atrioventricular block at the distal His bundle: Electrophysiological insights from left bundle branch pacing. HeartRhythm Case Rep. 2019;5(4):233-6

5. Huang W, Wu S, Vijayaraman P, et al. Cardiac resynchronization therapy in patients with nonischemic cardiomyopathy utilizing left bundle branch pacing. J Am Coll Cardiol EP 2020;(article in press)

6. Vijayaraman P, Sundaram S, Cano O, et al. Left bundle branch pacing for cardiac resynchronization therapy: Results from international LBBP collaborative study group. HRS 2020 - Late breaking clinical trial

7. Wang S, Wu S, Xu L, et al. Feasibility and efficacy of His bundle pacing or left bundle pacing combined with AV node ablation in patients with persistent atrial fibrillation and implantable cardioverterdefibrillator therapy. J Am Heart Assoc. 2019;17;8(24).e014253. doi 10.1161/JAHA 119.014253.
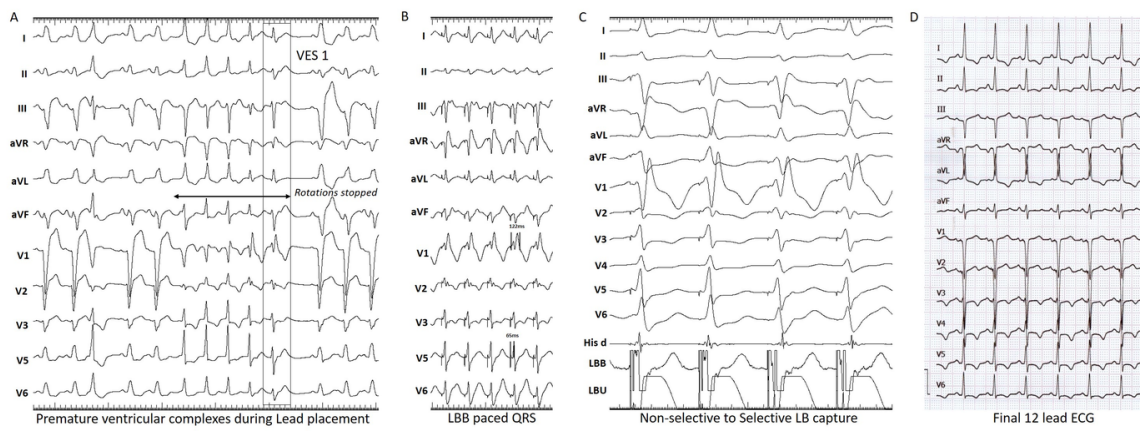

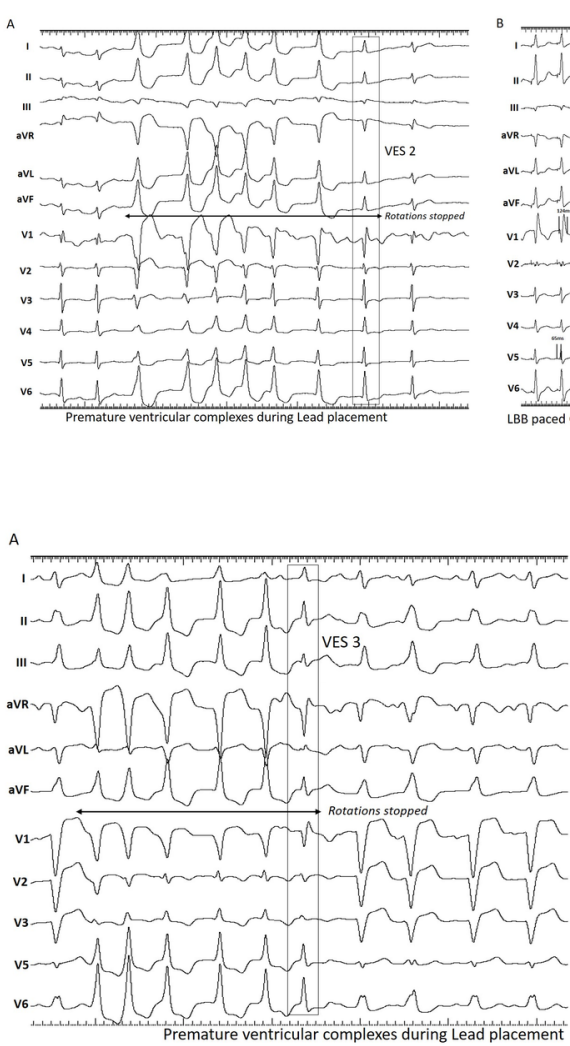

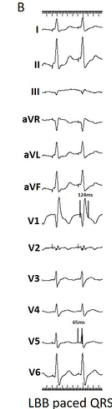

(n)

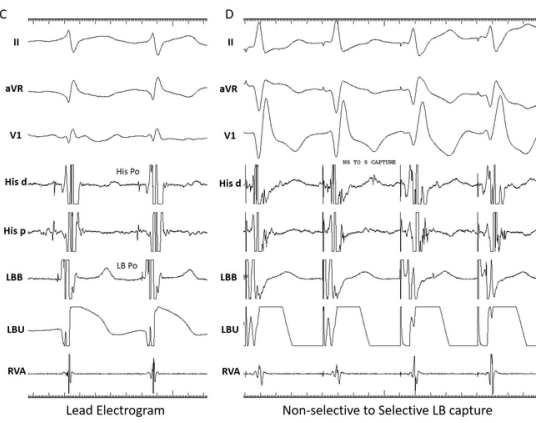

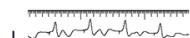

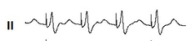

"1 Nortertir

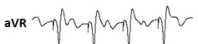

ave arurenterer

ave ath b th be

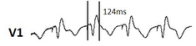

ve rmentrow

ve vithitwithr

v 20

vonturituter

LBB paced QRS

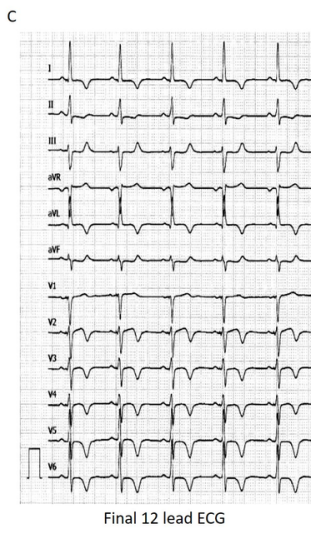

